\title{
WHOI SDSL Data-Link Project—Ethernet Telemetry through Sea Cables
}

\author{
Marshall Swartz, Daniel J. Torres, Steve Liberatore, AND Robert Millard \\ Woods Hole Oceanographic Institution, Woods Hole, Massachusetts
}

(Manuscript received and in final form 28 October 2011)

\begin{abstract}
A data telemetry technique for communicating over standard oceanographic sea cables that achieves a nearly 100-fold increase in bandwidth as compared to traditional systems has been recently developed and successfully used at sea on board two Research Vessel (R/V) Atlantis cruises with an 8.5-km, 0.322-in.diameter three-conductor sea cable. The system uses commercially available modules to provide Ethernet connectivity through existing sea cables, linking serial and video underwater instrumentation to the shipboard user. The new method applies Synchronous Digital Subscriber Line (SDSL) communications technology to undersea applications, greatly increasing the opportunities to use scientific instrumentation from existing ships and sea cables at minimal cost and without modification.
\end{abstract}

\section{Background}

Since the first use of conducting wire to telemeter data from instruments lowered from ships, users have faced significant constraints in both data speed and maximum achievable distance. Seemingly a technology plateau was reached, because for over three decades, various telemetry schemes achieved less than $10 \mathrm{kbit} \mathrm{s}^{-1}$ data rates over 10-km-long single- or three-conductor cables developed for oil drilling instrumentation and control applications. In the United States, the "standard CTD" sea cable is the Tyco/Rochester three-conductor instrumentation and control cable model A301592, consisting of three twisted inner copper conductors within a twolayer steel torque-balanced outer armor. This model cable has been employed for over 20 years and is aboard all Woods Hole Oceanographic Institution (WHOI) ships, as well as much of the University-National Oceanographic Laboratory System (UNOLS) fleet. Appendix C details the Rochester A301592 sea cable specifications and presents results from analyses of our test cable. Many new technology applications have been constrained by limitations on telemetry speeds over twisted triplet- or single-conductor armored cables. Those applications requiring greater bandwidth have been forced to employ

Corresponding author address: Marshall Swartz, Woods Hole Oceanographic Institution, 266 Woods Hole Road, MS 30, Woods Hole, MA 02543.

E-mail:mswartz@whoi.edu coaxial or fiber optic cables, with their higher cost, maintenance issues, and limited availability. Only three UNOLS ships carry fiber optic cables, and there are only two portable fiber optic winches presently available in the United States that permit such cable to be used on other vessels. Consequently, schemes to observe real-time video of the seafloor acquire instantaneous lowered acoustic Doppler current profiler (LADCP) data, or "sniff" for hydrothermal vent plumes using underwater mass spectrometers that have been shelved due to their inability to support the required data rates on traditional sea cables.

\section{SDSL technology}

Late in 2008, while setting up instrumentation aboard the Research Vessel (R/V) Knorr, we observed that an off-the-shelf Synchronous Digital Subscriber Line (SDSL; International Telecommunications Union 2003) telemetry system using two inner conductors of the Knorr's 9.4-km three-conductor sea cable was able to establish and maintain Ethernet connectivity at data rates far exceeding any previously observed. The telemetry system was a commercially available industrial SDSL product manufactured by Black Box, Inc., using one unshielded twisted-pair cable to extend Ethernet networks over long distances. The product, model LR0020A SDSL Network Extender kit, consists of a "provider" unit and a "subscriber" unit, one used at either end of the twistedpair cable. A derivative of the residential Internet digital 
subscriber line (DSL)-over-telephone line products, this model has been in production for over 8 years and has been used extensively in industrial applications (Dobrowski et al. 2002). SDSL technology uses many narrow-frequency channels, or bins, ranging from $4 \mathrm{kHz}$ to $2.6 \mathrm{MHz}$, to transmit data packets on the wire pair, automatically adapting to current conditions by choosing those bins that maximize data rates and minimize packet drop. The system uses a balanced line wire pair; we have not found operations on a single-conductor sea cable to be reliable.

There is substantial supporting documentation on the application and design of networks using this technology, but we have found no mention of use in underwater applications, such as sea cables. We contacted Black Box engineering staff to discuss our application, and while they had no recollection of such use, they saw no reason why it would not work.

\section{Laboratory testing}

We purchased a Black Box LR0020A SDSL kit for evaluation and testing at WHOI in late 2008. Connecting this system to a decommissioned 7.4-km-long A301592 sea cable kept at our laboratory for testing purposes, we found that the SDSL kit worked immediately. Using the SDSL pair to connect our test of the three-conductor sea cable from the gigabit WHOI local network to a PC with network test software, we achieved full-duplex Ethernet speeds exceeding $800 \mathrm{kbit} \mathrm{s}^{-1}$. These speed tests were verified using bandwidth-testing applications available on the Internet (see http://www.dslreports.com/ speedtest) and testing hardware from the WHOI Computer and Information Services group.

Subsequent tests in the laboratory confirmed uninterrupted and error-free data transfers of over $30 \mathrm{~GB}$ between the WHOI network and the test laptop using the SDSL set over the sea cable. Applications having "cable internet-rated" bandwidth requirements, such as video Skype, real-time CNN broadcasts, and real-time video camera uploads were also tested and shown reliable, with uninterrupted transmission lasting for days. Further testing demonstrated that the concurrent use of the SDSL Data-Link on two conductors with a SeaBird SBE911plus CTD operating on the third conductor with armor ground return is also viable. For this test, a standard SBE9plus CTD with SBE32 pylon was attached to one conductor and the cable armor, with a standard SBE11plus version 2 deck unit attached to the opposite end of the cable, applying $260 \mathrm{~V}$ to the ground to drive the CTD/pylon. This test was conducted to demonstrate that the SDSL Data-Link is able to operate at a reduced data rate on the two remaining sea cable conductors without modification to the usual singleconductor-to-armor connection employed on many UNOLS ships for Sea-Bird SBE911plus CTD systems. In this mode, the SDSL Data-Link demonstrated a reduced $400 \mathrm{kbit} \mathrm{s}^{-1}$ data rate, but neither system experienced any data losses. Crosstalk from the neighboring Sea-Bird differential phase shift keyed (DPSK) signal on the adjacent conductors is the likely explanation for the reduced data rate of the SDSL system because of the schemes that adapt its telemetry frequency span in the presence of noise.

\section{Real-time LADCP acquisition}

One of the first applications envisioned for the SDSL Data-Link involved concurrent real-time CTD and LADCP data acquisition over existing sea cables. The standard mode of LADCP operation (dual-instrument configuration) involves communicating with the ADCP instruments via a dedicated data cable connected directly to the instruments while the underwater package is on deck. Prior to a CTD cast, while powered from an external battery pack mounted on the underwater support frame, a command file is sent to each LADCP with the appropriate data collection commands. Subsequent data acquisition is carried out autonomously by the LADCP instruments. The problem with this procedure is there is no way to monitor the LADCP data collection or change any settings once the underwater package is deployed. In addition, the acquired data cannot be accessed for display and processing until after the cast has been completed and the data downloaded from the ADCP instruments.

Laboratory tests were conducted involving two Teledyne RD Instruments (RDI) Workhorse Sentinel (WHS)-300 LADCP units connected through the SDSL Data-Link while attached to the $7.4-\mathrm{km}$ test sea cable. Numerous tests proved conclusively that the SDSL Data-Link provided fully reliable and low-latency serial connection to allow for operation of the LADCP instruments in any desired mode. Serial RS232 connections were established and maintained for over $48 \mathrm{~h}$ with two operating WHS-300 LADCPs at 115200 baud.

\section{Ethernet rate tests}

Laboratory tests demonstrated that the SDSL telemetry system as described above provides an $800 \mathrm{kbit} \mathrm{s}^{-1}$ fullduplex Ethernet link through A301592 sea cables up to $8.5 \mathrm{~km}$ in length. Consistent Ethernet data rates of $400 \mathrm{kbit} \mathrm{s}^{-1}$ have been demonstrated when used together with the SBE911plus CTD system. 


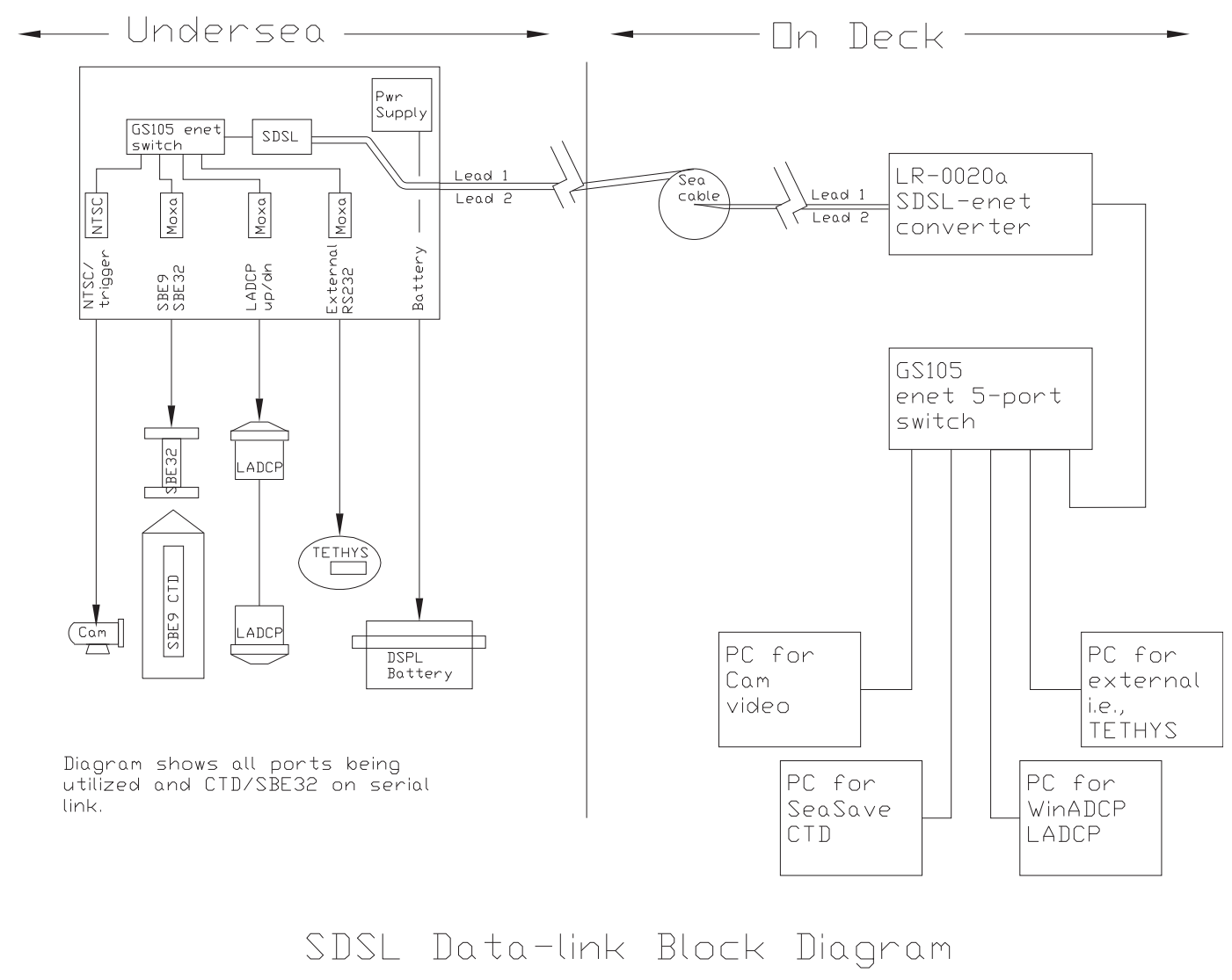

FIG. 1. The SDSL Data-Link prototype. System consists of the power supply unit; the SDSL Data-Link, which integrates CTD; a rosette water sampler; a pair of LADCPs (upward/downward looking); a mass spectrometer named TETHYS, and camera data.

\section{Configuration of prototype SDSL Data-Link}

Based on the successful preliminary tests, a prototype operational SDSL Data-Link system was built as shown in Fig. 1. It consists of two subsystems: an underwater unit and a topside unit. The prototype underwater unit consists of a direct current (DC) power supply, an SDSL-Ethernet adapter, a five-port Ethernet switch, three two-port serial-Ethernet adapters, and one video-Ethernet server. With the exception of the power supply, all components are commercially available devices. They are mounted on an aluminum chassis affixed to the blank end cap of an underwater housing. The opposite end cap has bulkhead connectors to provide the necessary connections to the sea cable, external battery, and external devices shown in appendix A. The Data-Link pressure case is made of cylindrical anodized aluminum 70-71 TG, 26.5 in. $(67.3 \mathrm{~cm})$ long (exclusive of connectors on one end), and $7.5 \mathrm{in} .(19.1 \mathrm{~cm})$ wide, and weighs approximately $20 \mathrm{~kg}$ in air and $12 \mathrm{~kg}$ in seawater. It has been pressure tested at WHOI to 6800 dbar.
The prototype Data-Link is intended to be mounted onto a CTD/rosette frame or a towed vehicle, such as the WHOI Towcam. The prototype's power supply is capable of providing regulated power to a SBE9plus CTD and an SBE32 water sampler pylon. Either a 24or $48-\mathrm{V}$ battery can power the Data-Link; the power supply automatically accommodates battery voltage ranging from 20 to $60 \mathrm{~V}$. If an LADCP system is to be used, then a $48-\mathrm{V}$ external battery is required to directly power the WHS-300 ADCPs while also supplying power to the Data-Link. Otherwise, a $24-\mathrm{V}$ battery is sufficient to power the Data-Link. If the SBE9plus CTD and SBE32 pylon are attached for use in serial mode, then these are also powered by the external battery. All other instruments connected to the Data-Link must be selfpowered.

The prototype system supports up to six concurrent RS-232 serial data ports at speeds up to $115 \mathrm{kbit} \mathrm{s}^{-1}$ and one analog [National Television System Committee (NTSC) or Phase Alternating Line (PAL)] video input. Also available is an internal Ethernet port, which can be wired to a bulkhead connector if required. It is not 
expected that all of these ports will be simultaneously used at their maximum data rate, because the SDSL Data-Link cannot handle the combined data from all ports running at maximum theoretical speed.

The top-side equipment consists of two small components. Two inner leads from the sea cable must be available in the science laboratory; these are connected to a second SDSL-Ethernet converter. A five-port gigabit Ethernet switch connects the SDSL-Ethernet converter with application computers for the various instruments. Appendix B shows the equipment in laboratory. Users must provide the computers required for the specific underwater instruments attached to the SDSL Data-Link. For serial devices connected to the SDSL Data-Link underwater unit, the computers must have a vendor-supplied software driver for the DataLink's serial-Ethernet converters. This makes the underwater serial-Ethernet adapter appear to be a local serial port of that computer. For other devices, such as the video-Ethernet converter, the application computer simply uses either a web browser or a video monitor application software package.

\section{Results of operation at sea}

Testing at sea was conducted in the late summer and fall of 2009, during two cruise legs of R/V Atlantis. The prototype quickly demonstrated its usefulness and operated successfully from the first trial. On the first cruise leg AT15-52, a mass spectrometer instrument named TETHYS (Camilli and Duryea 2009) was mounted onto the Atlantis rosette frame and connected to the SDSL Data-Link to enable real-time measurements of methane and other trace gas distributions in the water column. The SDSL Data-Link installation took approximately $10 \mathrm{~min}$ to complete. The system ran continuously from 14 through 27 September without failure. During that time, 26 consecutive stations were completed using the TETHYS mass spectrometer (operating at $38.4 \mathrm{kbit} \mathrm{s}^{-1}$ ). The data display and instrument control provided by the SDSL Data-Link allowed water samples to be collected at specific depths based on the real-time TETHYS chemical data. The total acquisition time for the 26 stations was less than $24 \mathrm{~h}$ and is composed of approximately 2000 measurement points. Later, on cruise AT15-54, the SDSL Data-Link was again used on the CTD/rosette frame, this time with a single RDI WHS-300 LADCP, and a Deep Sea Power and Light (DSPL) camera, with the intention of testing the real-time control and acquisition of the LADCP and video imagery. The SDSL Data-Link immediately passed all tests with both systems running simultaneously. The first test of the SDSL Data-Link with the LADCP involved testing the ability to monitor data collection in real time. A command file was used to deploy the LADCP at the start of the cast through the SDSL unit. As was done in the laboratory test, the data were then monitored using WinADCP software from Teledyne RDI. We were able to successfully acquire single ping $(\sim 1 \mathrm{~Hz})$ velocity profiles and monitor various instrument properties (pitch, roll, and heading) without any transmission errors for the duration of the cast. The next test explored the ability to change the deployment parameters midcast. Once the instrument was within range of the bottom $(\sim 150 \mathrm{~m})$, a new command file was sent to the instrument to turn on the bottom-track mode. (In this mode, so-called bottom-track pings are alternated with water-track pings.) The cast was then continued to the bottom and then back up to $150 \mathrm{~m}$ off the bottom. At that point, another command file was sent to turn off the bottom-track mode for the rest of the upcast. Because bottom-track pings do not measure a water velocity profile, they are only useful while the instrument is within range of the bottom. The ability to change parameters during the cast allows one to optimize data collection to obtain the best possible final absolute velocity profile. We were able to change the instrument parameters during the cast without problem. Because of weather concerns over the use of the towed camera system, the DSPL camera using the SDSL Data-Link became the focus of scientific operations on nine consecutive stations and delivered 10-s photo imaging of the targeted areas in the Gulf of California at 1820-m depth.

As noted above, existing RDI software packages BBTALK and WinADCP were used to control, acquire data, and monitor the LADCP instruments during these stations. Although revealing that WinADCP was inadequate for real-time scientific interpretation of the LADCP data stream, the test cases were sufficient to demonstrate that the instrument was behaving as expected and that the data were properly streaming up the sea cable. An important part of LADCP data processing involves merging CTD and LADCP data streams in postprocessing by matching the change in CTD pressure over time $(d P / d T)$ with the LADCP vertical velocity (Thurnherr 2008). However, resolving problems with clock drift or data gaps can become labor intensive. The SDSL hardware supports the ability to time stamp all available data streams passing through the system in real time. Although we do not yet have the software for simultaneously acquiring LADCP and SBE9 CTD data in one application, we believe that such tools can be developed now that the data can be made available in real time through the SDSL system. 


\section{Implementation risk from uncertain sea cable conditions}

The greatest challenge to successful use of the SDSL Data-Link is expected to be the uncertain condition of the many three-conductor sea cables on ships in the UNOLS fleet. Normal use of the SBE911plus CTD system requires only a single conductor with armor return, and thus the other conductors in the sea cable are typically considered redundant. In addition, seagoing technicians often do not have the proper equipment and training to perform thorough electrical testing of all three conductors of the sea cables, slip rings, and leadin cables necessary to ensure that the SDSL Data-Link telemetry system will operate properly.

The prototype SDSL telemetry system described here requires at least two of the three conductors of the entire sea cable system to have end-to-end continuity of less than $350 \mathrm{ohms}$, and insulation resistance readings in excess of 1 gigaohms to ground between adjacent conductors, as measured using a cable testing megohmmeter at 250-V rating. If the SBE911plus CTD is to be used in the "traditional" mode on the third conductor, then all three conductors must meet this test. These specifications are less than the minimum specifications of the A301592 model three-conductor sea cables when new (see appendix C), and thus it is not unreasonable to expect that with sufficient notice, each ship with this sea cable can pretest their systems to know beforehand whether there is a problem meeting these standards and thereby remedy the problem before the SDSL system will be used.

\section{Summary}

We have described a prototype underwater telemetry package providing Ethernet connectivity through conventional sea cables. The system employs an industrial product capable of providing very long distance highspeed bidirectional Ethernet telemetry over a standard three-conductor sea cable as is used aboard most U.S. research ships. We have successfully demonstrated how SDSL technology enables many high-data-rate applications, such as the use of spectrometers, multichannel optical sensors, magnetometers, and still-image video to be sent over existing three-conductor sea cables. Previously, these applications required fiber optic or coaxial sea cables to achieve the necessary bandwidth; or, as in the case of LADCP operations, it was simply not possible to monitor the data collection or change instrument settings. A prototype SDSL system and power supply has been constructed at WHOI composed primarily of commercial off-the-shelf devices, packaged inside a pressure-tested 6800-m housing for use on underwater frames and towed vehicles.

Successful system operation at sea on R/V Atlantis cruises AT15-52 and AT15-54 was demonstrated on 35 consecutive stations with the SDSL Data-Link and the SBE9/11/32 rosette/CTD operating concurrently on an 8.5-km-long UNOLS CTD cable, operating with the TETHYS mass spectrometer (26 stations) and the DSPL camera ( 9 stations) with LADCP (4 stations).

Acknowledgments. We thank Dr. Andrew Remsen of University of South Florida for bringing our attention to the use of the SDSL device for sea cable telemetry. Drs. Richard Camilli, Adam Soule, Daniel Lizarralde, and Dan Fornari have been strong supporters of this development and we thank them for their willingness to try this prototype on their cruises. We also thank Dr. John Toole for his constructive comments and support. Darryl Symonds of Teledyne RDI generously provided firmware upgrades in order to test and compare the various bottom-track features of the LADCP.

This development program has been supported, in part, through research grants from the National Science Foundation (OCE 0447395), the National Aeronautics and Space Administration's ASTEP program (NNX09AB76G), and a WHOI Green and Hiam Innovative Technology Award.

\section{APPENDIX A}

\section{Underwater Block Diagram}

Figure A1 illustrates the underwater block diagram.

\section{APPENDIX B}

\section{Top-Side Block Diagram}

Figure B1 illustrates the top-side block diagram.

\section{APPENDIX C}

\section{Rochester Model A301592 Instrumentation and Control Cable Performance Characteristics}

The latest specifications for this model sea cable (http://www.rochestercables.com/pdfs/DataLines/EOP/ A301592.pdf) are as follows:

Insulation resistance of 3000 megaohms km ${ }^{-1}$, conductor DC resistance of: $30.8 \mathrm{ohms} \mathrm{km}{ }^{-1}$, armor DC resistance of $7.9 \mathrm{ohms} \mathrm{km}{ }^{-1}$, and capacitance from the conductor to armor of 0.115 $\mu \mathrm{F} \mathrm{km}^{-1}$. 


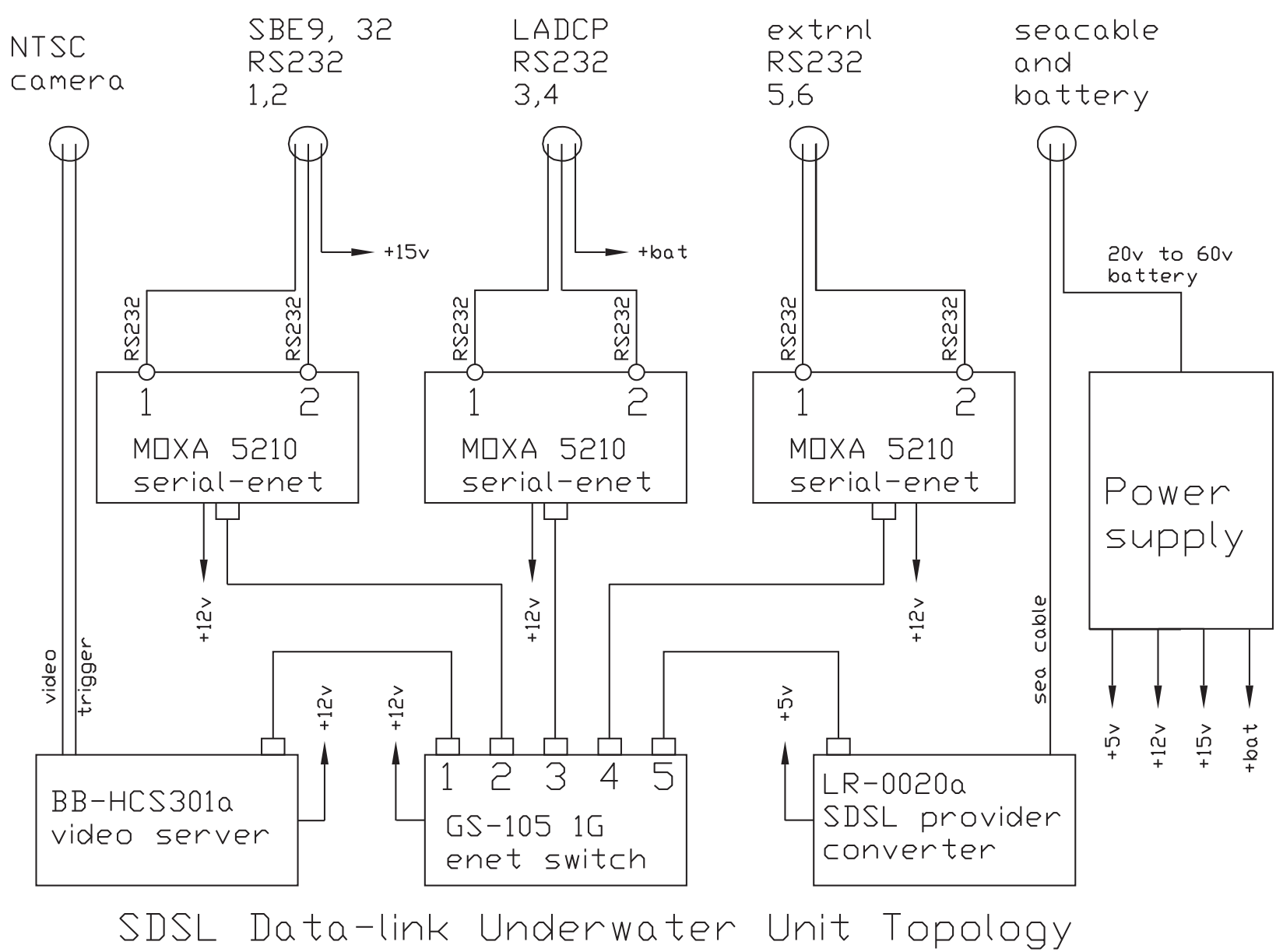

FIG. A1. The SDSL Data-Link prototype underwater unit. Details show major components mounted on the chassis.

\section{a. Characteristics of this model sea cable as tested on 17 May 2009 at WHOI}

The actual length of the tested sea cable is $7435 \mathrm{~m}$, as determined by the embedded marker tape. The armor shows moderate rusting and corrosion. This wire was first put into service about 1992 and removed from service about 1996.

All readings shown below were taken with 20-m-long American wire gauge (AWG) 16 lead-in cables attached on both ends.

Insulation characteristics of a conductor to other the two conductors tied to armor, as measured with $260 \mathrm{~V}$ applied for 10-min reading, are as follows:

Red: 1.95 gigaohms, polarization index of 0.91 , and capacitance of $0.91 \mu \mathrm{F}$ (red);

White-1: 2.14 gigaohms polarization index of 0.99 , and capacitance of $0.95 \mu \mathrm{F}$; and

White-2: 3.27 gigaohms, polarization index of 1.01, and capacitance of $0.95 \mu \mathrm{F}$.

\section{b. The conductor $D C$ resistance at $20^{\circ} \mathrm{C}$}

The conductor $\mathrm{DC}$ resistance at $20^{\circ} \mathrm{C}$ is as follows:

Red: 291.0 ohms,

White-1: $298.0 \mathrm{ohms}$,

White-2: $300.6 \mathrm{ohms}$, and

Armor: 64.2 ohms.

\section{c. Observations}

Observations from the study follow:

Insulation resistance of the conductors has fallen below specification likely resulting from aging, moisture penetration, and fatigue.

Actual capacitance of the tested sea cable equates to $0.13 \mu \mathrm{F} \mathrm{km}^{-1}$, which is about $13 \%$ higher than the specification.

Actual conductor resistances of all conductors are higher than the expected 228 ohms over a $7.4-\mathrm{km}$ length, likely resulting from work hardening. The 
PCs with ethernet NIC and Moxa Nport Administrator serial driver software.

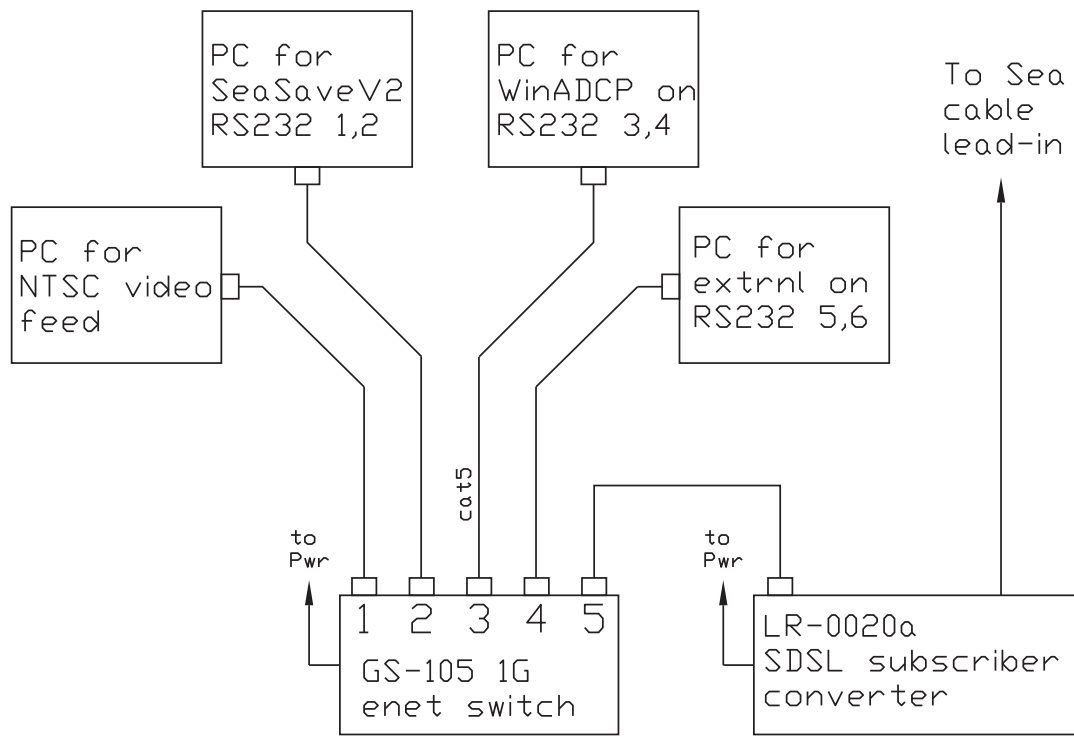

\section{SDSL Data-link Top-side Topology}

FIG. B1. The SDSL Data-Link components used in the top-side laboratory. Only two components are required: the SDSL converter and an Ethernet switch to connect the user-provided computers to the local network. Science users provide the necessary PCs with software drivers to acquire the serial or video data.

resistance contribution of the lead-in cables (approximately $0.25 \mathrm{ohms}$ per lead) has been subtracted from the DC resistance measurements shown above.

Attenuation by the actual test sea cable to signals in the frequency range used by the SDSL telemetry is expected to be higher than that for a new sea cable.

\section{d. Measurement instruments}

The measurement instruments are as follows:

Insulation resistance, polarization index, and capacitance readings were made with an AEMC model 1060 automated insulation tester/megohmmeter.

Conductor and armor DC resistance readings were taken with a Keithley model 2001 DMM in fourwire offset compensation resistance mode on the 2000-ohm range.

\section{REFERENCES}

Camilli, R., and A. N. Duryea, 2009: Characterizing spatial and temporal variability of dissolved gases in aquatic environments with in-situ mass spectrometry. Environ. Sci. Technol., 43, 5014-5021, doi:10.1021/es803717d.

Dobrowski, G. H., S. Mukherjee, J. Engstrom, S. Lindecke, A. Nicholson, and B. Dropping, 2002: Symmetric DSL white paper. DSL Forum, 15 pp. [Available online at http://www. broadband-forum.org/marketing/download/mktgdocs/SHDSL_ wp.pdf.]

International Telecommunications Union, 2003: Single-pair highspeed digital subscriber line (SHDSL) transceivers. Series G: Transmission systems and media, digital systems and networks: Digital sections and digital line system-Access networks, ITU-T Recommendation G.992.1, 190 pp. [Available online at http:// www.analytic.ru/articles/lib28.pdf.]

Thurnherr, A. M., 2008: How to process LADCP data with the LDEO software. User's manual, $30 \mathrm{pp}$. [Available online at ftp://ftp.ldeo.columbia.edu/pub/ant/LADCP/UserManuals/howto.pdf.] 